\title{
3D Correlative Microscopy for Real World Problem Solving
}

\author{
Bartlomiej Winiarski ${ }^{1}$, Adam Brinek ${ }^{2}$, Ali Chirazi ${ }^{1}$ and Daniel Lichau ${ }^{1}$ \\ ${ }^{1}$ Thermo Fisher Scientific, United States, ${ }^{2}$ Brno Uni of Technology / CEITEC, United States
}

Current scientific and industrial real world challenges span across different length scales and are driven by various physical and chemical phenomenon. The dawn of two- and three-dimensional (2D and 3D) correlative microscopy and tomography (CMT) greatly advanced understanding real world problems in life and material sciences [1-6]. 2D/3D CMT for the same regions of interest and at different length scales allows spatial and temporal registration in two and three dimensions of many imaging modalities. For example in life sciences, visible light imaging, electron and cathodoluminescence imaging is often coregistrated. In materials sciences, $\mathrm{x}$-ray computed tomography, electron and ion microscopy, electron backscattered diffraction (EBSD), energy-dispersive X-ray spectroscopy (EDS), wavelength-dispersive X-ray spectroscopy (WDS) analytics, magnetic resonance imaging, Raman, atomic force microscopy (AFM), secondary-ion mass spectrometry (SIMS), X-ray photoelectron spectroscopy (XPS), etc. information may be merged bringing a new insight on the same region of interest.

CMT in materials [1-5] and life sciences [6] uses number of micro/nano x-ray computed tomography $(\mu \mathrm{CT})$ scanners, optical microscopes, liquid metal ion source (LMIS) and Plasma DualBeam and Laser Plasma DualBeam microscopes (with analytics, e.g. EBSD, EDS, SIMS, etc.), transmission electron microscopes (TEM), nano computed tomography (nCT) in TEM and scanning electron microscope (SEM), etc. There are two key factors that makes CMT approachable and successful for real world problems: (a) interrogation volumes and probe size (resolutions) of various techniques must overlap (Fig. 1 ), and (b) coordinate systems of samples and region of interest (RoI) are tracked and registered in 2D and $3 \mathrm{D}$.

The advent of Plasma focused ion beams (FIBs) allowed bridging the gap between macro- and nano-scale and accessing region of interest buried few hundreds of microns below the surface [1]. While commercially available femtosecond Laser Plasma FIB [4, 7, 8] with ease can access locations below $1 \mathrm{~mm}$ in a dozen of minutes [8] keeping the sub-nanometer imaging resolution of SEM column.

Typically, apparatuses are self-contained and have different requirement for sample preparation, size, fixture, handling and transferring etc., thus direct sample transfer between is very limited or entirely prohibited. While commercially available correlative microscopy transfer means are designed for 2D data acquisition, e.g. optical microscopy (OM) to SEM, SEM to Raman, etc. with extension to, somewhat 3D, superficial surface layers (dozens of microns) when using LMIS FIB-SEM. The first real 3D correlative tomography and microscopy purpose designed cross-platform ( $\mu \mathrm{CT}$ to DualBeams) sample transfer and Maps ${ }^{\text {TM }}$-based correlation solution was recently proposed by Winiarski at al. [4] and is already adopted in academic and industrial environments.

In this contribution we focus on advancements of 3D correlative microscopy and workflows for real world problems solving, e.i. in battery research, jet engines, additive manufacturing, polymer composites, and semiconductor industries. In the studies we use set of research instruments, HeliScan $\mu \mathrm{CT}$, DualBeams and TEM microscopes and various cross-platform coordinates registration methods (e.g. in Fig. 2). The workflows we use achieve highest $\mu \mathrm{CT}$ resolution (below $400 \mathrm{~nm}$ ) and allow real 3D correlative tomography and microscopy by accessing region of interest in any location of the specimen and collecting quantitative information using 3D-EBSD/EDS, etc. Our correlative hardware solution is supported by 
Maps-based [4] and Avizo-based workflows (Fig. 2) [1] and is compatible with the inert gas transfer hardware and the cryo stage.

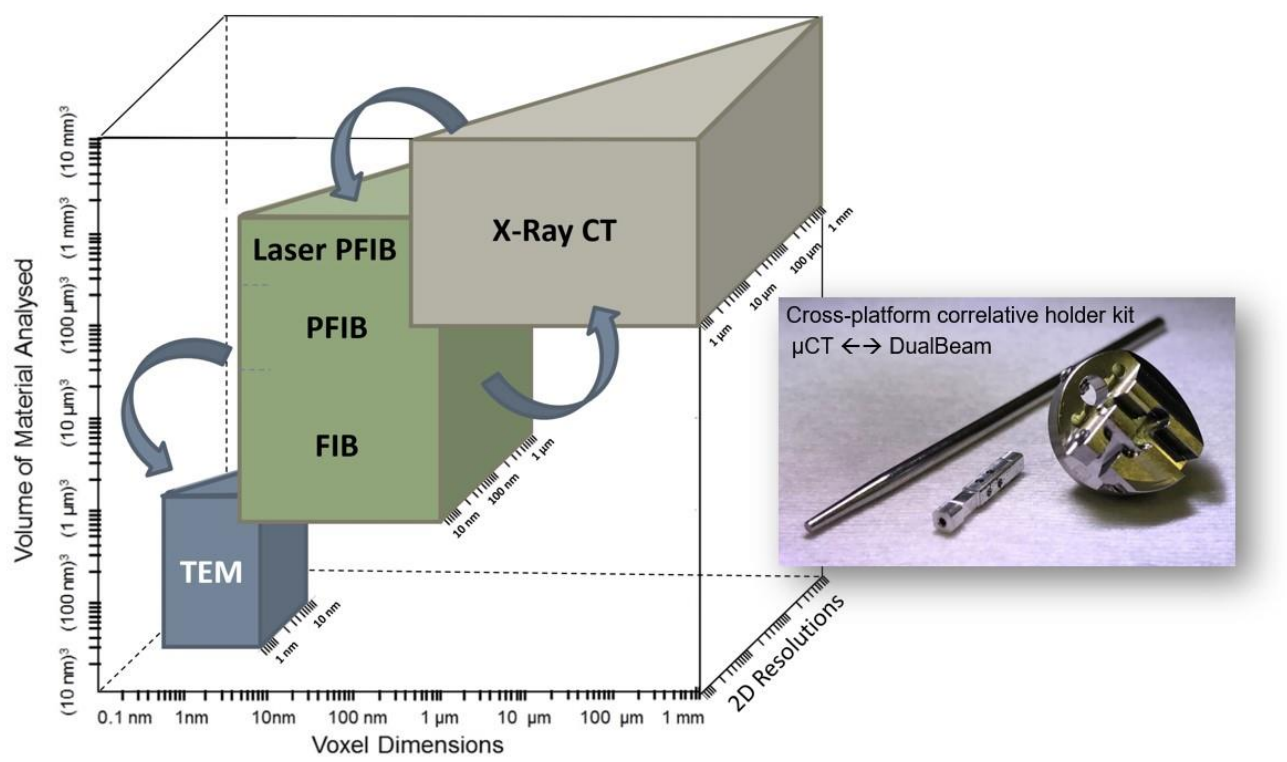

Figure 1. Shows multi-scale and multi-modal imaging methods typically used in materials science. DualBeam platforms are used both for sample preparation for other systems and for the data collection, where arrows show possible sample transfers and systems stage coordinates co-registration.
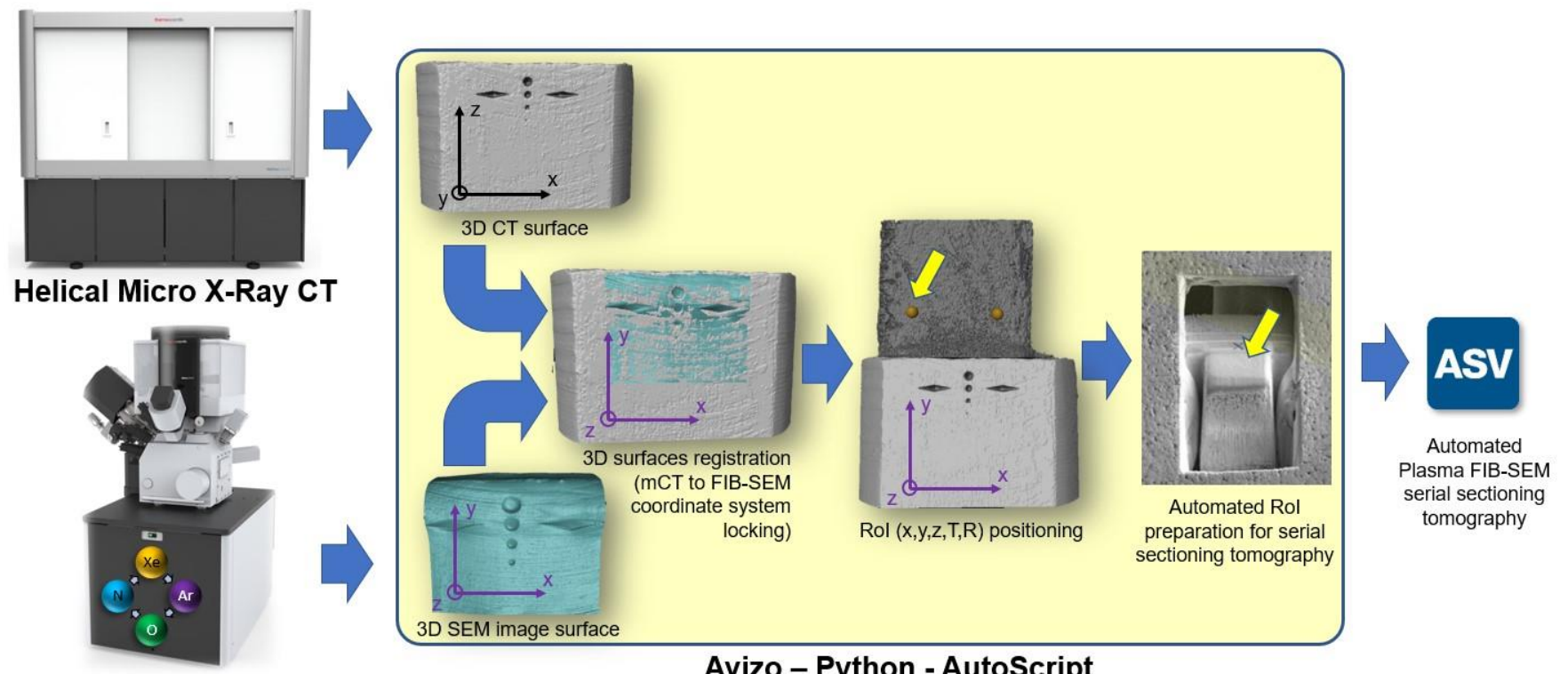

Plasma FIB-SEM

Avizo - Python - AutoScript

Figure 2. Shows Avizo/Python/AutoScript - based workflow for micro CT to DualBeam coordinate locking and automated site preparation for serial sectioning tomography.

\section{References}

[1] B Winiarski at al. Supplement of Microscopy and Microanalysis 152 (2017), p. S4-S9.

[2] B Winiarski at al. Microscopy and Microanalysis 23 (S1/2017), p. 342-343.

[3] TL Burnett et al. Scientific Reports 4 (2014), p. 4711.

[4] B Winiarski at al. Microscopy and Microanalysis 25(S2)-2019:870-871. 
[5] R Moroni, et al. Scientific Reports 6 (2016), p. 30109.

[6] S Meschini. European journal of histochemistry 2017 EJH 61(s4):1.

[7] SJ Randolph et al. Journal of Vacuum Science \& Technology B36 (2018), p. 06JB01.

[8] B Winiarski \& R Geurts. Wiley Analytical Science, Aug. 2020, https://analyticalscience.wiley.com/do/10.1002/was.00070019. 